
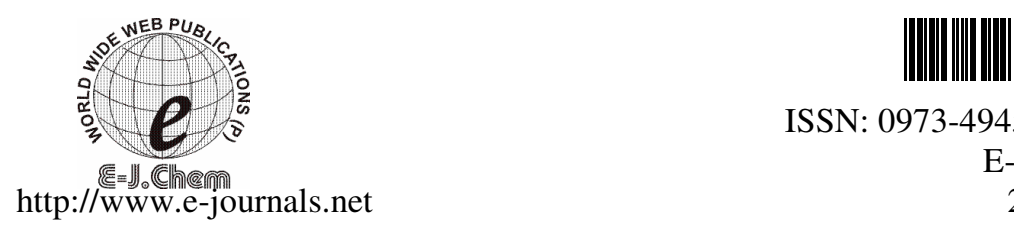

ISSN: 0973-4945; CODEN ECJHAO

E-Journal of Chemistry 2010, 7(S1), S23-S26

\title{
Effect of Molar Concentration on Optical Absorption Spectra of ZnS:Mn Nanoparticles
}

\author{
RAVI SHARMA*, B.P.CHANDRA ${ }^{\S}$ and D.P.BISEN \\ *Department of Physics, Arts \& Commerce Girls College \\ Devendra Nagar, Raipur (C.G.), 492001, India \\ ${ }^{\S}$ Department of Applied Physics, Shri Shankaracharya College of Engineering \& \\ Technology, Junwani Bhilai (C.G.) 490020, India \\ School of Studies in Physics, Pt. Ravishankar Shukla University \\ Raipur (C.G.) 492010, India \\ rvsharma65@gmail.com
}

Received 19 July 2009; Accepted 7 September 2009

\begin{abstract}
The present paper reports the synthesis and characterization of luminescent nanocrystals of manganese doped zinc sulphide. Nanocrystals of zinc sulphide were prepared by chemical precipitation method using the solution of zinc chloride, sodium sulphide, manganese chloride and mercaptoethanol was used as the capping agent. It was found that change in the molar concentration changes the particle size. The particle size of such nanocrystals was measured using XRD pattern and it is found to be in between $3 \mathrm{~nm}-5 \mathrm{~nm}$. The blue-shift in absorption spectra was found with reducing size of the nanoparticles
\end{abstract}

Keywords: Nanophosphor; XRD, Luminescence, Optical absorption spectra, ZnS:Mn.

\section{Introduction}

Semiconductor nanocrystals are described as a state of matter that is intermediate between individual molecule and bulk ${ }^{1}$. Transition from bulk to nanoparticles lead to the display of quantum mechanical properties and an increased dominance of surface atoms which increases the chemical reactivity of a material. Notable examples include tunable bandgap ${ }^{2}$ and catalytic behavior ${ }^{3}$, respectively. The small size and high optical activity of certain semiconductors make them interesting for applications in disciplines ranging from optoelectronics ${ }^{4}$, catalysis ${ }^{5}$ to fluorescence microscopy ${ }^{6}$.

Particles in nanometric sizes show unique physical properties, for example with the decrease of particle size, extremely high surface area to volume ratio is obtained leading to an increase in surface specific active sites for chemical reactions and photon absorption to enhance the reaction and absorption efficiency. The enhanced surface area increases surface 
states, which changes the activity of electrons and holes, affecting the chemical reaction dynamics. For instance, the size quantization can increase the bandgap of photocatalysts to enhance the redox potential of conduction band electrons and valence band holes ${ }^{7}$. Also, nanoparticles can induce the possibility of indirect electron transitions at the boundary of the crystals and realize the essential enhancement of light absorption.

The conventional approach for synthesis of nanoparticles involves chemical or physical attrition from bulk into objects of desired sizes and shapes (e.g. mechanical milling, ion implantation, etc.), and is referred as the 'top-down' approach. Inverse to the 'top-down' approach is a process universal in nature, involving the assembly of materials from molecular levels to form micro or macro-sized shapes and structures, often referred as 'bottom-up' approach or 'self-assembly' .

$\mathrm{ZnS}$ is semiconducting materials ${ }^{9,10}$, which has a wide band gap of $3.70 \mathrm{eV}$. In this work zinc sulfide nanocrystals are prepared by chemical precipitation technique and mercaptoethanol has been used for capping, which modifies surface of nanoparticles and prevents the growth of the particles to larger size. The effect of concentration of mercaptoethenol on the particle size, effect of changes in Mn concentrations on optical absorption spectra and the change in the molar concentration of the reactants on the particle size has been investigated.

\section{Experimental}

The most important step in the studies of nanoparticles is their synthesis. There are various methods supported for synthesis of nanoparticles. Chemical route was used in the present investigation. The powder of $\mathrm{ZnS}$ nanoparticles were prepared by using chemical deposition technique described by Khosravi ${ }^{11}$. For synthesis, $1 \mathrm{M}$ aqueous solution of $\mathrm{ZnCl}_{2}$ and $1 \mathrm{M}$ aqueous solution of $\mathrm{Na}_{2} \mathrm{~S}$ were mixed in the presence of various concentration of mercaptoethenol solution. $\mathrm{MnCl}_{2}$ was also mixed in the solution in ratio 99:1, while stirring the solution continuously. The obtained precipitate was washed thoroughly three to four times in double distilled water and then separated by centrifuge at $3500 \mathrm{rpm}$ and finally air dried. Special care was taken to maintain the same physical condition during the synthesis of the sample.

All the samples were characterized at Inter University Consortium (IUC) Indore. The morphologies and sizes of the mercaptoethanol capped ZnS:Mn were determined by X-ray diffraction studies with $\mathrm{Cu} \mathrm{K} \alpha$ radiation $(\lambda=1.5418 \AA$ ) . XRD data were collected over the range $20^{0}-70^{\circ}$ at room temperature. X-ray diffraction patterns have been obtained by Rigaku Rotating Anode (H-3R) diffractometer. The particle size was calculated using the Debye-Scherrer formula.

Absorption spectra of the samples prepared with various concentrations of capping agent were studied. Perkin Elimer $\lambda-12$ spectrometer was used to obtain the absorption spectra of $\mathrm{ZnS}: \mathrm{Mn}$ nanoparticles.

\section{Results and Discussion}

The XRD patterns for the samples are shown in Figure 1.Three different peaks are obtained at $2 \Theta$ values of $29.50^{\circ}, 48.80^{\circ}$ and $57.80^{\circ}$. This shows that the samples have zinc blende structure and the peaks correspond to diffraction at (111), (220) and (311) planes, respectively ${ }^{12}$. The lattice parameter has been computed as $5.31 \AA$, which is very close to the standard value (5.42 $\AA$ ). It is also seen from the Figure 1 that peaks are broadened for higher concentration of capping agent. The broadening of peaks indicates nanocrystalline behavior of the particles. The size of the particles has been computed from the width of first peak using Debye Scherrer formula ${ }^{13}$. 

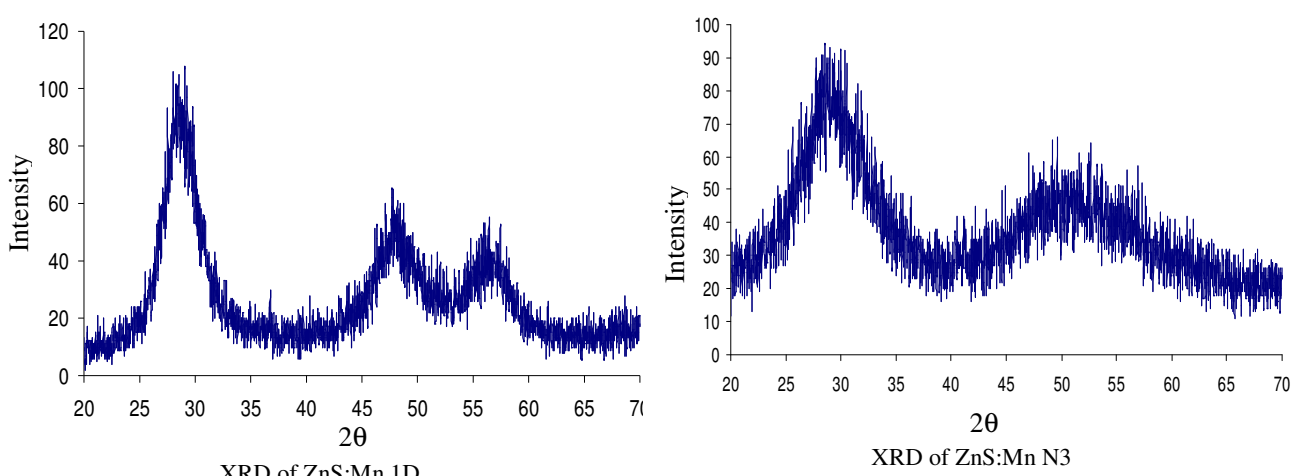

Figure 1. XRD pattern of $\mathrm{ZnS}: \mathrm{Mn}$ with molar concentration $1 \mathrm{M}$ and $10^{-2} \mathrm{M}$

The XRD spectra of the ZnS:Mn nanoparticles with different molar concentration of the reactants are different . It is clear from the figure that the peak width is less for the samples with higher molar concentration $(1 \mathrm{M})$ to that of the lower molar concentration $\left(10^{-2} \mathrm{M}\right)$. The particle size of the sample for $1 \mathrm{M}$ concentration is $4.16 \mathrm{~nm}$ and $3.46 \mathrm{~nm}$ for $10^{-2} \mathrm{M}$ was observed.

Figure 2(a) shows the optical absorption spectra of nanoparticles with different concentration of mercaptoethanol. It was found that with the increasing concentration of mercaptoethanol the peak shifts towards the lesser value of wave length. The observed blue shift in the absorption edge is reflection of the band gap increase owing to quantum confinement effect. In the bulk material the band gap can be found from the graph between $\left(\alpha h v^{2}\right) v s . h v$. whereas in the nanomaterials the band gap is obtained from the absorption maxima. The band gap energy of the samples corresponding to the absorption edge was found in the range $4.5 \mathrm{eV}-4.88 \mathrm{eV}$ with increasing the capping agent concentration. Similar results were reported by Kumbhojkar et al. ${ }^{14}$ and Ruby et al. ${ }^{15}$.

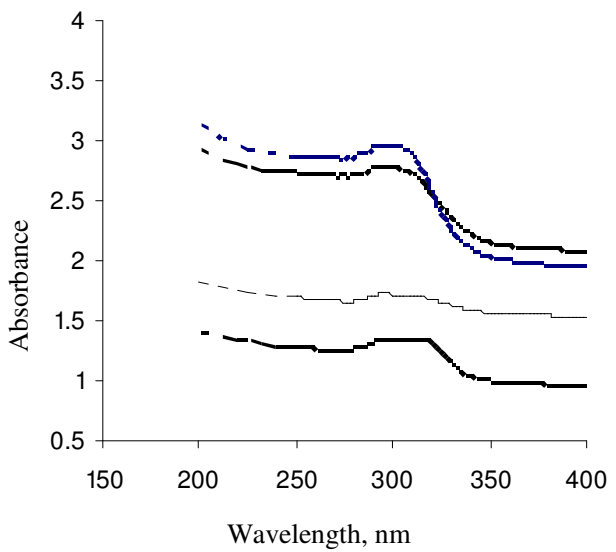

Figure 2(a). Optical absorption spectra with difference concentration of mercaptoethanol

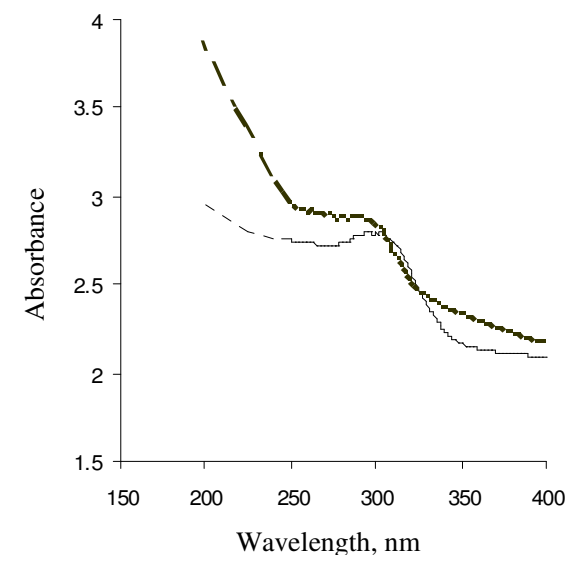

Figure 2(b). Optical absorption spectra of $\mathrm{ZnS}: \mathrm{Mn}$ of different molar concentration

Figure 2(b) shows the optical absorption spectra of the samples with different molar concentration of the reactants whereas the concentration of capping agent is unaltered. It is clear from the figure that the peak of the samples with lower molar concentration $\left(10^{-2} \mathrm{M}\right)$ shows the shift towards the lesser wave length to that of the samples with higher molar 
concentration $(1 \mathrm{M})$. It is also clear that optical absorption edge is showing blue shift for the samples with lower molar concentration to that of the samples with higher molar concentration. The effective mass approximation (EMA) model predicting the variation of exciton energy with particle size is used to estimate the crystalline size. It is seen that particle size estimated from absorption edge is of the same order as that obtained from broadening of XRD peaks. Increase in band gap corresponds to the smallest dimension of the nanocrystals, where as XRD corresponds to average size. Nearly same values of particle size by effective band gap and XRD reveal that nanoparticles are spherical in nature.

\section{Conclusion}

The nanoparticles of ZnS:Mn were grown by chemical routs in which mercaptoethanol was used as capping agent . The XRD pattern indicated the growth of the nanoparticles. The size of nanoparticle was found to decrease with increasing concentration of mercaptoethanol. The measurement of optical absorption edge indicates the reduction in particle size with the decrease in the molar concentration of reactants.

\section{Acknowledgment}

All the samples were characterized at Inter University Consortium (IUC) Indore. The first author is very much thankful to Dr. R.J. Choudhary of Inter University Consortium (IUC) Indore for providing XRD facility. One of the author (RS) is also very much thankful to U.G.C. India for funding this work partly as minor research project.

\section{References}

1. Cyr P W, Tzolov M, Hines M A., Manners I, Sargent E H and Scholes G D, J Mater Chem., 2003, 13, 2213-2219.

2. Fendler H and Meldrum F C, Adv Mater., 1995, 7, 607-632.

3. Lopez N, Janssens T V W, Clausen B S, Xu Y, Mavrikakis M, Bligaard T and Norskov J K, J Catal., 2004, 223, 232-235.

4. Alivisatos A P, Science, 1996, 271, 933-937.

5. Ahmadi T S, Wang Z L, Green T C, Henglein A and El-Sayed M A, Science, 1996, 272, 1924-1926.

6. Yelin D, Oron D, Thiberge S, Moses E and Silberberg Y, Opt Express, 2003, 11, 1385-1391.

7. Hoffman A J, Mills G, Yee H and Hoffmann M R, J Phys Chem., 1992, 96, 5546-5552.

8. Dutta $\mathbf{J}$ and Hofmann $\mathrm{H}$, Self-organization of colloidal nanoparticles in: H.S. Nalwa (Ed.), Encyclopedia of Nanoscience and Nanotechnology, American Scientific Publishers, California, 2004, 617-640.

9. Denzler D, Olschewski M and Sattler K, J Appl Phys., 1998, 84(5), 2841.

10. Zou B S, Little R B, Wang J P and E L-Sayed M A, Int J Quant Chem., 1999, 72, 439-450.

11. Khosravi A A, Kundu M, .Shekhawat G S, Gupta R R, Sharma A K, Vyas P D and Kulkarni S K, Appl Phys Lett., 1995, 67, 2506.

12. Mahamuni S, Khosravi A A, Kundu M, Kshirsagar A, Bedekar A, Avasare D B, Singh P and Kulkarni S K, J Appl Phys., 1993, 73(10), 5237-5240.

13. Guinier A, X-ray Diffraction, Freeman, San Francisco, 1963.

14. Kumbhojkar N, Nikesh V V, Kshirsagar A and Mahamuni S, J Appl Phys., 1999, 88(11), 6260.

15. Tamrakar R, Ramrakhiani M and Chandra B P, Open Nanoscience J., 2008, 2, 12-16. 


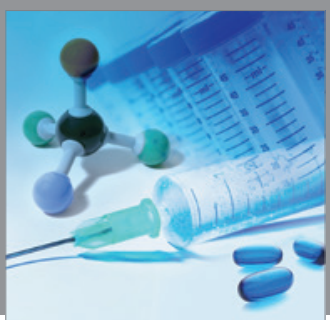

International Journal of

Medicinal Chemistry

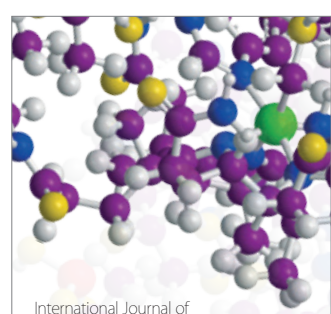

Carbohydrate Chemistry

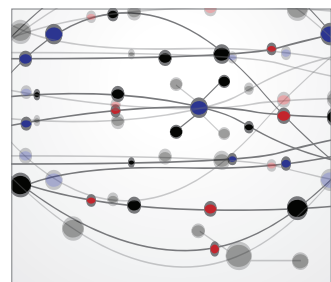

The Scientific World Journal
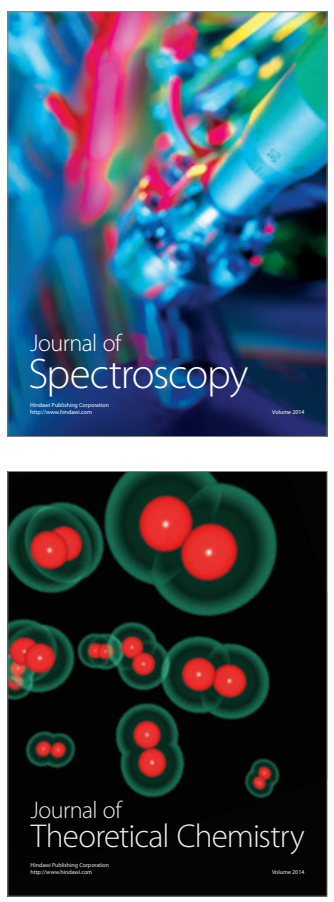
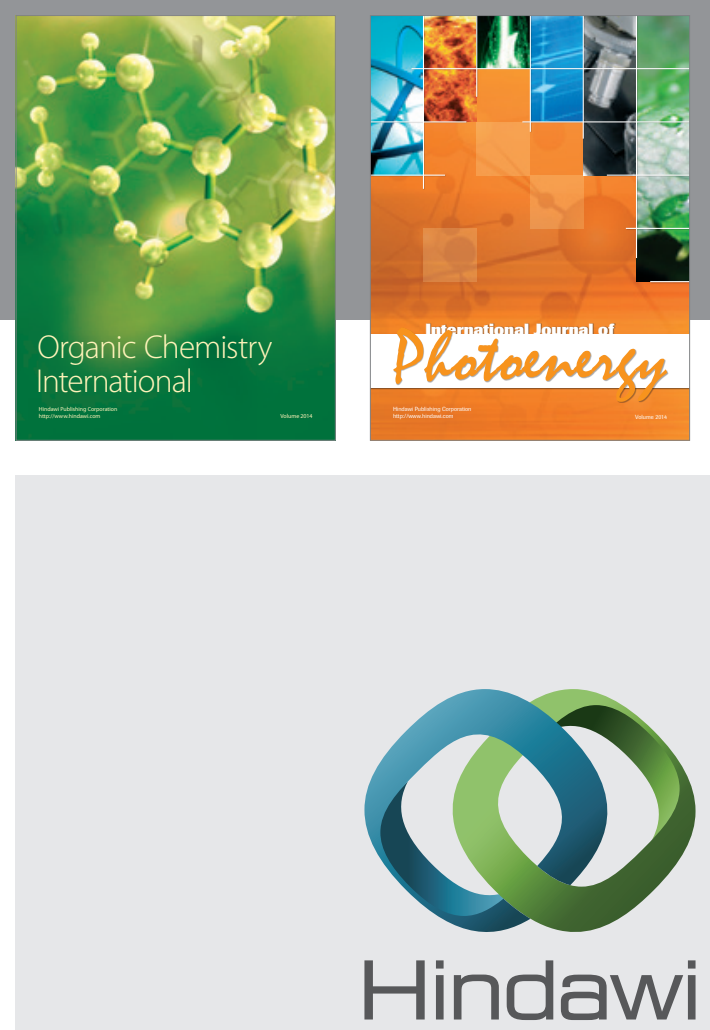

Submit your manuscripts at

http://www.hindawi.com
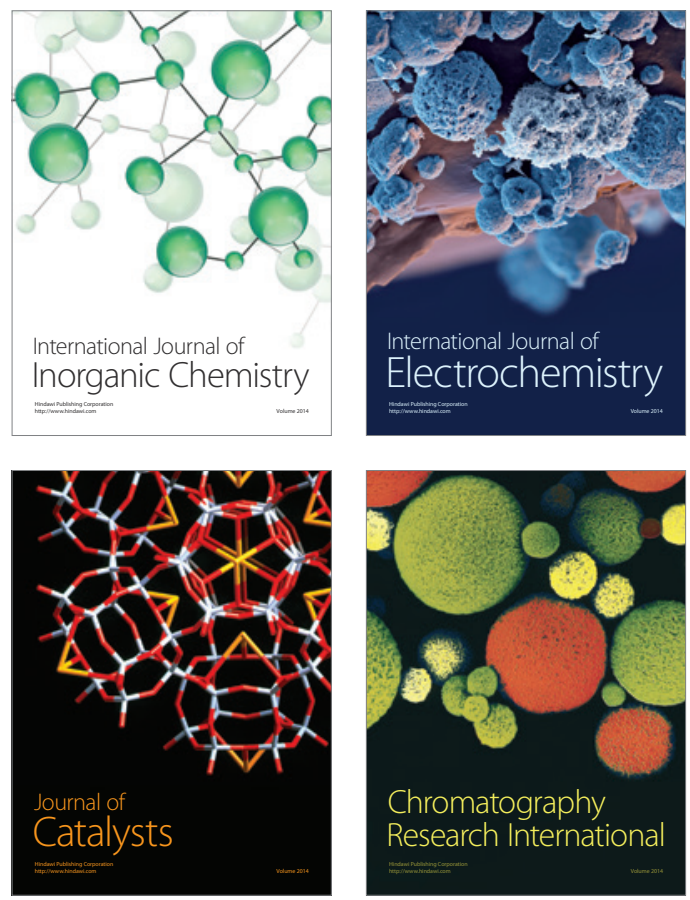
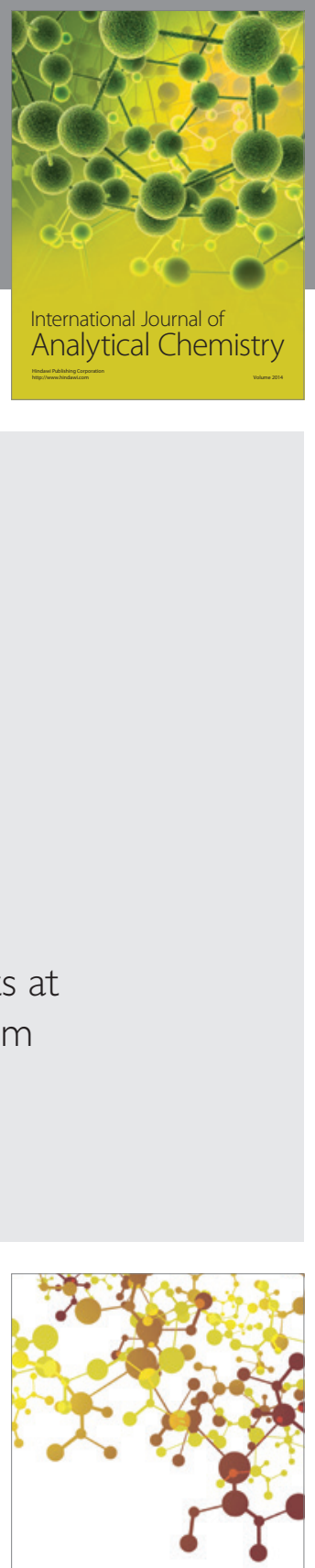

Journal of

Applied Chemistry
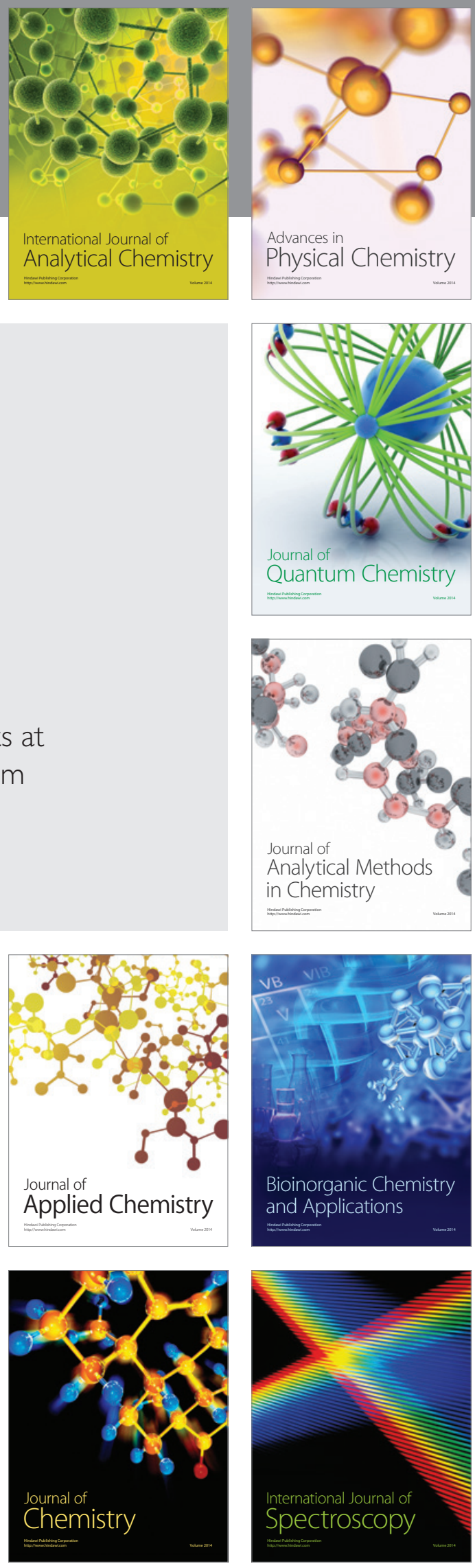